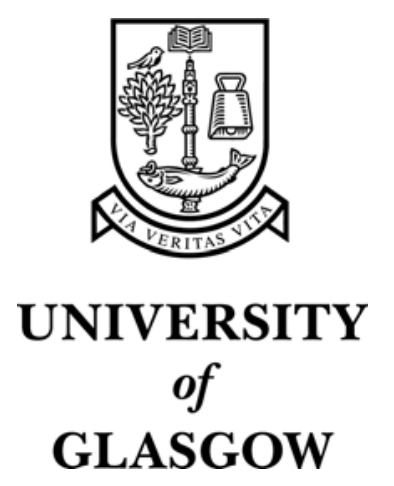

Jones, W.P. and Paul, M.C. (2005) Combination of DOM with LES in a gas turbine combustor. International Journal of Engineering Science 43(5-6):pp. 379-397.

http://eprints.gla.ac.uk/3327/ 


\title{
Combination of DOM with LES in a Gas Turbine Combustor
}

\author{
W. P. Jones ${ }^{1}$ and M. C. Paul ${ }^{2}$ * \\ ${ }^{1}$ Department of Mechanical Engineering, Imperial College London, \\ Exhibition Road, London SW7 2AZ, UK \\ ${ }^{2}$ Department of Mechanical Engineering, University of Glasgow, \\ Glasgow G12 8QQ, UK
}

\begin{abstract}
A three-dimensional numerical study is conducted to investigate the radiative heat transfer in a model gas turbine combustor. The Discrete Ordinates Method $\left(\mathrm{DOM} / S_{n}\right)$ has been implemented to solve the filtered Radiative Transfer Equation (RTE) for the radiation modelling and this has been combined with a Large Eddy Simulation (LES) of the flow, temperature and composition fields within the combustion chamber. The radiation considered in the present work is due only to the hot combustion gases notably carbon dioxide $\left(\mathrm{CO}_{2}\right)$ and water vapour $\left(\mathrm{H}_{2} \mathrm{O}\right)$, which is also known as the "nonluminous' radiation. A benchmark problem of the ideal furnace is considered first to examine the accuracy and computational efficiency of the DOM in the three-dimensional general body fitted co-ordinate systems.
\end{abstract}

Keywords: Discrete Ordinates Method, Large Eddy Simulation, Radiative Heat Transfer, Turbulent Flow, Combustion

\section{Introduction}

In most gas turbine combustors a large part of the heat transfer to and from the walls of the combustion chambers occurs by radiation. This radiation has two components: (i) the 'non-luminous', which emanates from the combustion gases notably carbon dioxide $\left(\mathrm{CO}_{2}\right)$ and water vapour $\left(\mathrm{H}_{2} \mathrm{O}\right)$, and (ii) the 'luminous', which is mainly due to the soot formed in the flame.

The prediction of wall temperatures is an important aspect in the design of practical engine combustors and this clearly requires that the radiative heat fluxes be predicted accurately. An inability to predict the wall temperatures may lead to an excessive amount of the combustor airflow being used for cooling the liner wall and this is likely to lead to a reduced combustion efficiency and an increased emission

\footnotetext{
${ }^{*}$ Corresponding author: E-mail:m.paul@mech.gla.ac.uk, Tel:+44 (0)141 330 8466, Fax:+44 (0)1413304343
} 
of the pollutants such as carbon monoxide $(C O), N O_{x}$ formations and unburned hydrocarbons $(U H C)$. In addition, excessive combustor wall temperatures have a deleterious impact on combustor 'life'.

Chandrasekhar [1] first proposed a method, known as the Discrete Ordinates Method (DOM), in his work on one-dimensional stellar and atmospheric radiation. Subsequently Carlson and Lathrop [2] developed the DOM for multidimensional radiation problems employing the finite volumes approach. More recently, the DOM has been widely used on various different problems $[3 ; 4 ; 5 ; 6 ; 7]$ where the major emphasis has been on solving the Radiative Transfer Equation (RTE), which is the steady state representation of the radiative transfer. The radiative transfer in hightemperature combustion devices requires a simultaneous solution of the RTE and the governing flow equations such as Navier-Stokes, enthalpy and species concentrations conservation equations, etc $[8 ; 9 ; 10]$.

With respect to combining the RTE with the LES, only a little work has been done to date to the authors' knowledge. Recently, Desjardin and Frankel [10] have studied soot formation in the near field of a strongly radiating turbulent jet flame involving LES and a simplified two-dimensional treatment of radiation involving gray and non-scattering medium. Here, our interest is a combining the three-dimensional form of the RTE with the LES. While this requires a large amount of computer resource essentially because of the integro-differential nature of the RTE, parallel computation with today's CPU speeds allow a time-accurate and efficient simulation in this regard. The DOM is found to be the best suited method for domain based parallelism compared with the other methods such as Discrete Transfer (DT), Finite Volume (FV) and Finite Element (FE) methods [11; 12].

The first objective of this paper is to develop an efficient three-dimensional numerical Discrete Ordinates Method, for solving the Radiative Transfer Equation in a general body-fitted coordinate system. The second objective is to incorporate this method into a Large Eddy Simulation of flow, temperature and composition fields, and finally apply the devised methods to a gas turbine combustion chamber to investigate the radiative heat transfer.

\section{Physical and mathematical models}

This section describes the physical model and geometry of the model gas turbine combustor, followed by the descriptions of the filtered governing conservative equations for the radiative transfer and the Large Eddy Simulation of flow and combustion. In LES the large-scale turbulent motions are resolved while the small-scale turbulence is modelled $[13 ; 14 ; 15]$. The small-scale or subgrid-scale (SGS) modelling is described in Section 2.3. The Section 2.4 describes the details of the modelling and the properties of radiation, and the required boundary conditions to solve the radiative transfer equation.

\subsection{Physical model and geometry}

Fig. 1 shows the main features of the model gas turbine combustor, which is representative of the Rolls-Royce Tay gas turbine [16]. The combustor walls are made of 
transply, a laminated porous material. The geometry of the combustor includes a relatively small swirler at the head of the combustor in the centre of which the fuel injector is located and a hemispherical head section attached to a circular barrel of $75 \mathrm{~mm}$ diameter. This barrel contains a set of six primary ports/holes of $10 \mathrm{~mm}$ diameter each at the front and another set of six dilution ports/holes of $20 \mathrm{~mm}$ diameter each at $80 \mathrm{~mm}$ downstream of the first set. A circular-to-rectangular nozzle is attached to the end of the barrel. High purity gaseous fuel comprising over $95 \%$ propane $\left(\mathrm{C}_{3} \mathrm{H}_{8}\right)$ was injected into the combustion chamber through the centre of the swirler.

\subsection{Governing equations}

The equations of motion in LES may be obtained by applying a spatial filter, a mechanism to separate the large-scale (resolved) variables from the small scales. The filtered value of a generic variable $\phi\left(x_{j}, t\right)$ is defined, [17], as its convolution with a filter function, $G$, according to:

$$
\bar{\phi}\left(x_{j}, t\right)=\int_{\Omega} \phi\left(x_{j}, t\right) G\left(x_{j}-x_{j}^{\prime}, \Delta\left(x_{j}\right)\right) d x_{j}^{\prime},
$$

where $\Omega$ is the entire flow domain and $\Delta\left(x_{j}\right)$ is the filter width.

Large density variations occur in turbulent reacting flows and this must be properly accounted for. In LES the resolved scale density variations are calculated explicitly whilst the subgrid scale density variations are treated by the introduction of a density weighted Favre type filter, [18]. Favre-filtered quantities are denoted by ( .) and are defined as:

$$
\tilde{\phi}\left(x_{j}, t\right) \equiv \frac{\overline{\rho \phi}}{\bar{\rho}} .
$$

An application of the density weighted filter defined in Eq. (2) to the continuity, the Navier-Stokes, the mixture fraction, and the radiative transfer (discrete ordinates representation) equations gives:

$$
\begin{gathered}
\frac{\partial \bar{\rho}}{\partial t}+\frac{\partial \bar{\rho} \tilde{u}_{j}}{\partial x_{j}}=0 \\
\frac{\partial \bar{\rho} \tilde{u}_{i}}{\partial t}+\frac{\partial \bar{\rho} \widetilde{u_{i} u_{j}}}{\partial x_{j}}=-\frac{\partial \bar{p}}{\partial x_{i}}+\frac{\partial}{\partial x_{j}}\left(2 \mu \bar{S}_{i j}-\frac{2}{3} \mu \bar{S}_{k k} \delta_{i j}\right) \\
\frac{\partial \bar{\rho} \tilde{f}}{\partial t}+\frac{\partial \bar{\rho} u_{j} f}{\partial x_{j}}=\frac{\partial}{\partial x_{j}}\left(\frac{\mu}{P r} \frac{\partial \bar{f}}{\partial x_{j}}\right) \\
\alpha_{m} \frac{\partial \bar{I}_{m}}{\partial x}+\beta_{m} \frac{\partial \bar{I}_{m}}{\partial y}+\gamma_{m} \frac{\partial \bar{I}_{m}}{\partial z}+\overline{\left(\kappa+\sigma_{s}\right) I_{m}}=\overline{\kappa I_{b}}+\frac{\overline{\sigma_{s}}}{4 \pi} \sum_{m^{\prime}=1}^{M} \omega_{m^{\prime}} I_{m^{\prime}} \Phi_{m m^{\prime}}
\end{gathered}
$$

where $\rho$ is the mixture density, $t$ is the time, $x_{j}=(x, y, z)$ is the coordinate vector, $u_{j}$ is the velocity vector, $p$ is the dynamic pressure, $\mu$ is the coefficient of viscosity, $S_{i j}$ is the stain rate, defined as $S_{i j}=\frac{1}{2}\left(\frac{\partial u_{i}}{\partial x_{j}}+\frac{\partial u_{j}}{\partial x_{i}}\right), \delta_{i j}$ is the Kronecker delta, $f$ is the conserved scalar or mixture function and $\operatorname{Pr}$ is the Prandtl number. 
In Eq. (6), $I_{m}$ is the directional radiative intensity along the direction $\hat{s}_{m}$, where $m=1,2, \ldots, M$ (see Fig. 2) and the equation represents a set of $M$ different directional radiative intensities from each of the computational nodes. The subscripts $m$ and $m^{\prime}$ denote the outgoing and the incoming directions respectively and the terms $\alpha_{m}, \beta_{m}$ and $\gamma_{m}$ in Eq. (6) represent the direction cosines of the discrete direction $\hat{s}_{m}$ along the coordinates (see Fig. 2) [19]. $I_{b}$ is the blackbody intensity at the temperature of the medium which is defined as $\frac{\sigma \tilde{T}^{4}}{\pi}$ where $\sigma$ is the Stefan-Boltzmann constant and $\tilde{T}$ is the temperature, $\kappa$ is the absorption coefficient, $\sigma_{s}$ is the scattering coefficient, $\omega_{m^{\prime}}$ is the quadrature weight corresponding to the direction $\hat{s}_{m^{\prime}}$ [19], and $\Phi_{m m^{\prime}}$ is the scattering phase function which determines the probability of a ray scattering from one direction $\hat{s}_{m^{\prime}}$ into another direction $\hat{s}_{m}$ (see Fig. 2).

\subsection{Subgrid-scale modelling}

The Favre-filter introduces unknown terms $\bar{\rho} \widetilde{u_{i} u_{j}}$ in Eq. (4) due to the non-linearity of the convective terms and leaves the equation unclosed. It is usual to define these terms as $[20]$

$$
\bar{\rho} \widetilde{u_{i} u_{j}}=\bar{\rho} \tilde{u}_{i} \tilde{u}_{j}+\tau_{i j}
$$

where $\tau_{i j}$ is unknown and is referred to as the residual or subgrid-scale stress, which must be modelled. The oldest and probably the most widely used model is that of Smagorinsky model [21] and this is used here; it is an eddy viscosity model of the form

$$
\tau_{i j}-\frac{1}{3} \delta_{i j} \tau_{k k}=-2 \mu_{s g s} \tilde{S}_{i j}
$$

where $\tilde{S}_{i j}$ is the Favre filtered strain rate and $\mu_{s g s}$ is the subgrid scale eddy viscosity. This eddy viscosity is given by:

$$
\mu_{s g s}=\bar{\rho} C_{s}^{2} \Delta^{2}\left|\tilde{S}_{i j}\right|
$$

where $\left|\tilde{S}_{i j}\right|=\left(2 \tilde{S}_{i j} \tilde{S}_{i j}\right)^{1 / 2}$ and $\Delta$ is the filter width, defined as $\Delta=\left(\Delta_{x} \Delta_{y} \Delta_{z}\right)^{1 / 3}$, is proportional to the local mesh spacing. The Smagorinsky constant, $C_{s}$, takes the typical value of around 0.1.

The mixture fraction equation (5) also contains the unknown term $\bar{\rho} \widetilde{u_{j} f}$. This is defined in terms of a subgrid scale scalar flux, $J_{j}$, viz

$$
\widetilde{\rho} \widetilde{u_{j} f}=\bar{\rho} \tilde{u}_{j} \tilde{f}+J_{j} .
$$

It is usual to employ a gradient model for this flux of the form [22]

$$
J_{j}=-\frac{\mu_{s g s}}{P r_{s g s}} \frac{\partial \tilde{f}}{\partial x_{j}}
$$

where $P r_{\text {sgs }}$ is the subgrid scale Prandtl/Schmidt number which is assigned a value of 0.7 in the present work.

The conserved scalar model is used for combustion and the dependence of species concentrations, temperature and density on the mixture fraction is obtained from the computations of an essentially unstrained laminar flamelet. The subgrid scale 
fluctuations are accounted for via a beta probability density function (PDF). Further details of this model are given in [15].

The radiative transfer equation (6) also contains some unknown terms, $\overline{\left(\kappa+\sigma_{s}\right) I_{m}}$, $\overline{\kappa I_{b}}$, etc, which are the nonlinear correlations between turbulence and radiation. In the present study the subgrid scale turbulence-radiation interactions are neglected; future studies are required to incorporate those interactions and to investigate their effects. Based upon the preliminary assumption made, the unknown terms in Eq. (6) are simply expressed as

$$
\overline{\left(\kappa+\sigma_{s}\right) I_{m}}=\left(\bar{\kappa}+\bar{\sigma}_{s}\right) \bar{I}_{m}, \quad \overline{\kappa I_{b}}=\bar{\kappa} \bar{I}_{b} .
$$

\subsection{Radiation modelling}

For radiation modelling in the gas turbine combustor, it is assumed that the enclosure contains an absorbing-emitting, non-scattering and radiatively gray medium. If the presence of the scattering (i.e., if $\sigma_{s} \neq 0$ in Eq. (6)), is considered the RTE will be coupled with both the incoming and the outgoing radiative intensities inside the medium. To calculate a single directional radiative intensity from a computational node it is required first the calculation of all scattered intensities into that node point, i.e., the incoming radiations, be calculated. This becomes computationally a very expensive calculation. The assumption of a non-scattering medium in the work is made for computational reasons but the medium is highly dominated by the absorption and the emission. Based on this assumption, the Eq. (6) takes the form:

$$
\alpha_{m} \frac{\partial \bar{I}_{m}}{\partial x}+\beta_{m} \frac{\partial \bar{I}_{m}}{\partial y}+\gamma_{m} \frac{\partial \bar{I}_{m}}{\partial z}=\bar{\kappa} \bar{I}_{b}-\bar{\kappa} \bar{I}_{m}
$$

\subsubsection{Absorption coefficient}

In gas turbine combustion chambers, the radiating species are typical the combustion products. The notably combustion products are $\mathrm{H}_{2} \mathrm{O}$ and $\mathrm{CO}_{2}$ along with the smaller amount of $\mathrm{CO}, \mathrm{H}_{2}$ and other minor species. At a high temperature, the spectral bands from $\mathrm{H}_{2} \mathrm{O}$ and $\mathrm{CO}_{2}$ are the most dominant feature in non-luminous radiation compared to other species [23]. Therefore, the absorption coefficient is based on a mixture of $\mathrm{H}_{2} \mathrm{O}$ and $\mathrm{CO}_{2}$ and is expressed as [8;24]

$$
\bar{\kappa}=0.1\left(\tilde{Y}_{\mathrm{H}_{2} \mathrm{O}}+\tilde{Y}_{\mathrm{CO}_{2}}\right) \quad\left(m^{-1}\right)
$$

where $\tilde{Y}_{\mathrm{H}_{2} \mathrm{O}}$ and $\tilde{Y}_{\mathrm{CO}_{2}}$ correspond to the mole fractions of $\mathrm{H}_{2} \mathrm{O}$ and $\mathrm{CO}_{2}$ respectively.

\subsubsection{Boundary conditions to solve the RTE}

Consider the combustor walls are diffusely emitting and reflective, and the appropriate boundary conditions required to solve the radiative transfer equation (13) are then

$$
\bar{I}_{m}=\epsilon_{w} \bar{I}_{b w}+\frac{1-\epsilon_{w}}{\pi} \sum_{\substack{m^{\prime}=1 \\ \alpha_{m^{\prime}}<0}}^{M} \omega_{m^{\prime}}\left|\alpha_{m^{\prime}}\right| \bar{I}_{m^{\prime}} \quad: x=-L_{x} / 2
$$




$$
\begin{array}{lc}
\bar{I}_{m}=\epsilon_{w} \bar{I}_{b w}+\frac{1-\epsilon_{w}}{\pi} \sum_{\substack{m^{\prime}=1 \\
\alpha_{m^{\prime}}>0}}^{M} \omega_{m^{\prime}}\left|\alpha_{m^{\prime}}\right| \bar{I}_{m^{\prime}} & : x=L_{x} / 2 \\
\bar{I}_{m}=\epsilon_{w} \bar{I}_{b w}+\frac{1-\epsilon_{w}}{\pi} \sum_{\substack{m^{\prime}=1 \\
\beta_{m^{\prime}}<0}}^{M} \omega_{m^{\prime}}\left|\beta_{m^{\prime}}\right| \bar{I}_{m^{\prime}} & : y=0 \\
\bar{I}_{m}=\epsilon_{w} \bar{I}_{b w}+\frac{1-\epsilon_{w}}{\pi} \sum_{\substack{m^{\prime}=1 \\
\beta_{m^{\prime}}>0}}^{M} \omega_{m^{\prime}}\left|\beta_{m^{\prime}}\right| \bar{I}_{m^{\prime}} & : y=L_{y} \\
\bar{I}_{m}=\epsilon_{w} \bar{I}_{b w}+\frac{1-\epsilon_{w}}{\pi} \sum_{\substack{m^{\prime}=1 \\
\gamma_{m^{\prime}}<0}}^{M} \omega_{m^{\prime}}\left|\gamma_{m^{\prime}}\right| \bar{I}_{m^{\prime}} & : z=-L_{z} / 2 \\
\bar{I}_{m}=\epsilon_{w} \bar{I}_{b w}+\frac{1-\epsilon_{w}}{\pi} \sum_{\substack{m^{\prime}=1 \\
\gamma_{m^{\prime}}>0}}^{M} \omega_{m^{\prime}}\left|\gamma_{m^{\prime}}\right| \bar{I}_{m^{\prime}} & : z=L_{z} / 2
\end{array}
$$

In Eqs. (15)-(20), the first terms on the right hand side are the outgoing radiative intensities from the surfaces while the second terms are the incoming radiative heat fluxes which are related to the incoming radiative intensities on the surfaces and also known as an irradiation. Here, $\bar{I}_{b w}=\frac{\sigma \tilde{T}_{w}^{4}}{\pi}$ is the black body intensity on the combustor walls at the temperature $\tilde{T}_{w}$ and $\epsilon_{w}$ is the walls emissivity.

\section{Numerical procedures}

In this section the numerical procedures used to solve the filtered governing equations (3)-(5) and (13) are described. The filtered equations are rewritten in general boundary/body fitted coordinates system using the approach introduced by Thompson [25], where the governing differential equations in the Cartesian coordinates are transformed into the curvilinear coordinates system. The details of the numerical procedures in the LES approach to solve Eqs. (3)-(5) have already been presented in $[26 ; 27]$ and will not be repeated. Here, attention is focused only on the procedure for solving the radiative transfer equation (13) along with the boundary conditions given in Eqs. (15)-(20). These are presented in the next section.

\subsection{Discrete Ordinates Method $\left(S_{n}\right)$}

\subsubsection{Description of the method}

After the coordinate transformation, the RTE in equation (13) may be rewritten in the curvilinear coordinates system as

$$
\sum_{i=e, n, r} \bar{I}_{m}^{i} S_{m}^{i}-\sum_{i=w, s, l} \bar{I}_{m}^{i} S_{m}^{i}=J^{P}\left(\bar{\kappa} \bar{I}_{b}-\bar{\kappa} \bar{I}_{m}\right)^{P},
$$

where the terms $S_{m}^{i}$ in Eq. (21) represent as

$$
S_{m}^{i}=\left(\alpha_{m} A_{x \xi}+\beta_{m} A_{y \xi}+\gamma_{m} A_{z \xi}\right)^{i} \quad i=e, w
$$




$$
\begin{array}{ll}
S_{m}^{i}=\left(\alpha_{m} A_{x \eta}+\beta_{m} A_{y \eta}+\gamma_{m} A_{z \eta}\right)^{i} & i=n, s \\
S_{m}^{i}=\left(\alpha_{m} A_{x \zeta}+\beta_{m} A_{y \zeta}+\gamma_{m} A_{z \zeta}\right)^{i} & i=r, l
\end{array}
$$

where $(\xi, \eta, \zeta)$ are the general curvilinear coordinates; $J$ is the Jacobian of the coordinate transformation; $A_{x \xi}, A_{y \xi}, A_{z \xi}$, etc are the cofactors of the Jacobian $J$; the superscripts e, w, etc indicate that the values are taken at the eastern, western, etc control volume surfaces respectively; and the superscript $P$ represents the value at the central node of the control volume (see Fig. 3).

To close the above system of Eqs. (21)-(24) relations are required between the radiative intensities on the control volume surfaces and the nodal intensities (see Fig. 3). Most often a linear relation is applied:

$$
\bar{I}_{m}^{P}=d \bar{I}_{m}^{e}+(1-d) \bar{I}_{m}^{w}=d \bar{I}_{m}^{n}+(1-d) \bar{I}_{m}^{s}=d \bar{I}_{m}^{r}+(1-d) \bar{I}_{m}^{l}
$$

where $0.5 \leq d \leq 1$, known as the "weighted diamond differencing" scheme proposed by Carlson and Lathrop [2]. A diamond difference or symmetric scheme corresponds to the value, $d=0.5$, which is the second order accurate central difference approach but is found to be unstable. The scheme gives positive-negative oscillatory values of the radiative intensities which are physically unrealistic [28]. Fiveland [3] suggested that if the dimensions of the control volumes were kept within a range such as $d \xi<\frac{|\alpha|_{\min }}{\bar{\kappa}(1-d)}, d \eta<\frac{|\beta|_{\min }}{\bar{\kappa}(1-d)}$, etc, then the negative intensities might be minimised but not totally avoided. Therefore, it becomes important to employ a negative intensity 'fixup' procedure such that when a negative intensity arises, the value of $d$ will be switched to 1.0 from 0.5 [7], or gradually increase the value of $d$ from 0.5 to 1.0 until a stable positive solution is achieved [8].

The simulations/computations were initially started employing the negative intensity 'fixup' procedures. However, it was found that due to the very complex shape of the combustor geometry those procedures produced physically unrealistic solutions of the RTE in the non-orthogonal computational grid and that negative intensities could not be totally avoided. Thus above 'fixup' procedures for negative intensities are only suitable for orthogonal type grid computations or if the computational/physical geometry is very simple $[7 ; 8]$. Therefore, an alternative suggested for complex geometries is the step scheme [6;29] whereby the downstream surface intensities are set equal to the upstream nodal intensities. Although this is a first order accurate approach, no negative intensities occurred in the computation. Based on use of the step scheme, the final discretised equation for the radiative transfer may be rewritten explicitly as

$$
a_{m}^{P} \bar{I}_{m}^{P}=a_{m}^{E} \bar{I}_{m}^{E}+a_{m}^{W} \bar{I}_{m}^{W}+a_{m}^{N} \bar{I}_{m}^{N}+a_{m}^{S} \bar{I}_{m}^{S}+a_{m}^{R} \bar{I}_{m}^{R}+a_{m}^{L} \bar{I}_{m}^{L}+b_{m}^{P},
$$

where the intensities with the superscripts E, W, etc, denote the values at the eastern, western, etc, nodal intensities and the coefficients are defined as

$$
\begin{gathered}
a_{m}^{P}=\sum_{i=e, n, r} \max \left(S_{m}^{i}, 0\right)-\sum_{i=w, s, l} \min \left(S_{m}^{i}, 0\right)+J^{P} \bar{\kappa}, \\
a_{m}^{I}=-\min \left(S_{m}^{i}, 0\right) \quad i=e, n, r \quad \text { and } \quad I=E, N, R, \\
a_{m}^{I}=\max \left(S_{m}^{i}, 0\right) \quad i=w, s, l \quad \text { and } \quad I=W, S, L,
\end{gathered}
$$

and

$$
b_{m}^{P}=J^{P} \bar{\kappa} \bar{I}_{b} .
$$




\subsubsection{Selection of the discrete ordinate directions}

The Discrete Ordinates Method or $S_{n}$, where $n$ represents the order of approximation, is based on a discrete representation of the directional variation of the radiative intensity. In a three-dimensional enclosure, the total number of the different discrete directions, $M$, to be considered at each computational node is again related to the order of the $S_{n}$ approximation and is defined as $M=n(n+2)[19 ; 2]$. For example, in the $S_{4}$ approximation of the DOM the radiative intensities in a total of $M=24$ discrete directions are calculated from each computational node point $P$ : in the case of $S_{6}$ total number of directions is $M=48$ while for $S_{8}$ it is $M=80$.

Only positive values of the direction cosines/ordinates and the associate weights are given in Table 1 and these cover one eighth (the first quadrant) of the total directions and the total range of the solid angles $4 \pi$, as the three-dimensional enclosure has eight corners. To cover the entire solid angles $4 \pi$, any or all values of $\alpha_{m}$, $\beta_{m}$ and $\gamma_{m}$ in the table may become positive or negative [2]. Therefore, each row of the table contains values of the direction cosines and the weights in total eight different directions.

\subsection{Solution algorithm}

Temperature and the absorption coefficient are calculated first to obtain the radiation sources and the boundary conditions. Without the presence of scattering, the RTE in Eq. (26) is uncoupled with the incoming radiative intensities and solved independently. The solution of the RTE proceeds with an initial guess of the radiative intensities along all the possible directions and starts with a global iterative process. At each iteration, the discretised Eq. (26) is solved in every direction of $\hat{s}_{m}$ and the boundary conditions in Eqs. (15)-(20) are updated for the next iteration. The new solutions are then replaced by the previous iterative solutions and this process continues until the following convergent condition is satisfied

$$
\max _{1 \leq m \leq M}\left|\bar{I}_{m}^{P(i+1)}-\bar{I}_{m}^{P(i)}\right| \leq 10^{-4}
$$

where $i$ in the number of iteration.

\section{Results and discussion}

The results of the application of the above described numerical method to an ideal furnace and the gas turbine combustor are presented in this section. A benchmark problem, the ideal furnace, is considered first to examine the accuracy and computational efficiency of the DOM in three-dimensional general body fitted co-ordinates and the results are compared with the results available in the literature.

\subsection{Ideal furnace - a test case}

The three-dimensional ideal furnace of Menguc and Viskanta [30] is chosen to validate the numerical method for radiative heat transfer. Fig. 4a shows a schematic diagram of the idealised furnace which is filled with an absorbing-emitting $(\sigma=0)$ 
gray gas with $\bar{\kappa}=0.1 \mathrm{~m}^{-1}$. The radiative transfer equation (26) is iteratively solved together with the following steady state energy equation with an internal heat source of $\nabla \cdot \overline{\mathbf{q}}=5 k W m^{-3}$

$$
\nabla \cdot \overline{\mathbf{q}}=\bar{\kappa}\left(4 \pi \bar{I}_{b}-\int_{4 \pi} \bar{I} d \Omega\right) .
$$

The temperatures on the six boundaries of the furnace and their emissivities are given as

$$
\begin{array}{lcl}
z=0: & T=1200 K, & \epsilon_{w}=0.85 ; \\
z=L_{z}: & T=400 K, & \epsilon_{w}=0.70 ; \\
\text { others }: & T=900 K, & \epsilon_{w}=0.70 .
\end{array}
$$

Fig. 4b shows the non-orthogonal body-fitted grid on $x-y$ plane. Although the ideal furnace has a regular three-dimensional geometry, a non-orthogonal grid is generated to this problem to test the numerical method for radiative transfer in body-fitted co-ordinate system. It is noted that the grid in the $z$-direction is uniform and perpendicular to the $x-y$ plane. In this computation, the DOM is applied with the grid nodes of $20 \times 20 \times 40$ in the $x, y$ and $z$ directions respectively.

Fig. 5 shows the comparisons of the temperature profiles and the net wall radiative heat flux distributions obtained by the various $S_{n}$ approximations such as $S_{4}, S_{6}$ and $S_{8}$ of the discrete ordinates method in non-orthogonal (NOR) grid; the $S_{4}$ approximation of the DOM in orthogonal (OR) grid; and the zone method obtained by Menguc and Viskanta [30]. In Fig. 5a, the temperature distributions are presented along the $x$-axis in three different $z$ locations at $y=1 \mathrm{~m}$ of the ideal furnace. As the horizontal location, $z$, increases, the temperature inside the furnace decreases and these predictions from the DOM in NOR grid are found to be a very good agreement with the DOM in OR grid and the zone method. In Fig. 5b, the radiative heat losses at hot wall $(z=0 \mathrm{~m})$ and gain at cold wall $(z=4 \mathrm{~m})$ at the centre line $(y=1 m)$ are presented, and which also show that for both walls the results obtained by the DOM with the NOR grid are in very good agreement with those of the other methods.

\subsection{Results in the gas turbine combustor}

In previous section, a test case was presented where the accuracy and efficiency of the discrete ordinate method in a general body-fitted non-orthogonal grid was discussed. This numerical method is now applied to the model gas turbine combustor.

The numerical grid employed in the simulation consists of a total of 96000 cells with $40 \times 60 \times 40$ grid nodes in the $x, y$ and $z$ directions respectively, and Table 2 shows the necessary parameters used in the present work. The computational results presented in Figs. 6-9 are at 65000 time steps, which is at the real clock time of $t \approx 0.036 \mathrm{sec}$. The emissivity of the combustor walls are kept at $\epsilon_{w}=0.1$. To reduce the computational load, the radiation results are obtained only by the $S_{4}$ approximation of the DOM.

Instantaneous results of the temperatures and the mole fractions of $\mathrm{CO}_{2}$ and $\mathrm{H}_{2} \mathrm{O}$ are plotted in Fig. 6(a-c), respectively, at various horizontal locations of the combustor. It is noted that these results are obtained first without considering the 
effect of radiation. As an input of the radiation sources, the measurement of both the temperature and the gaseous species is required, because the black body intensity, $\bar{I}_{b}$, is a function of the temperature and the absorption coefficient, $\bar{\kappa}$, plotted in Fig. 6d is considered to be a function of both the mole fractions of $\mathrm{H}_{2} \mathrm{O}$ and $\mathrm{CO}_{2}$. di Mare et al [15] compared the computational results of $\tilde{T}, \tilde{Y}_{\mathrm{H}_{2} \mathrm{O}}$ and $\tilde{Y}_{\mathrm{CO}_{2}}$ with that of the experimental measurements done by Bicen et al [16] and found a good agreement. The details of the turbulent flow and combustion characteristic inside the combustor were also discussed by those authors [15] and will not be repeated here. The main objective of the present paper is to investigate the radiative heat transfer which occurs from the combustion gases (notably for $\mathrm{H}_{2} \mathrm{O}$ and $\mathrm{CO}_{2}$ ) to the liner wall and vice versa. The relevant results are presented below.

Fig. 6(e-g) show the results of the radiation quantities at the same time step and the same horizontal locations as in Fig. 6(a-d). Fig. 6e shows that the total radiative intensity, $\bar{I}=\sum_{m=1}^{M} \bar{I}_{m}$, attains a maximum at the region where both the temperature and the absorption coefficient are maximum. Therefore, it provides clear evidence that the medium is highly dominated by the hot $\mathrm{H}_{2} \mathrm{O}$ and $\mathrm{CO}_{2}$ gases. The radiative heat flux vectors, $\overline{\mathbf{q}}$, calculated from

$$
\overline{\mathbf{q}}=\int_{4 \pi} \bar{I} \hat{s} d \Omega \approx \sum_{m=1}^{M} \omega_{m} \bar{I}_{m} \hat{s}_{m},
$$

show that the radiation transport is directed to the centre of the combustor from the high temperature and the high emitting-absorbing regions where the value of absorption coefficient, $\bar{\kappa}$, is also maximum (see Fig. 6f). Fig. $6 \mathrm{~g}$ shows contours of the magnitude of the radiative heat fluxes defined as $|\overline{\mathbf{q}}|=\left(\overline{\mathbf{q}}_{x}^{2}+\overline{\mathbf{q}}_{z}^{2}\right)^{1 / 2}$. As the length of each of the radiative flux vectors is proportional to its magnitude, these contours also show that the largest radiative flux vectors are located near the region of maximum temperature, $\tilde{Y}_{\mathrm{CO}_{2}}$ and $\tilde{Y}_{\mathrm{H}_{2} \mathrm{O}}$.

Fig. 7 shows the net loss or gain of the energy due to the radiation as a divergence of the radiative heat fluxes, $\nabla \cdot \overline{\mathbf{q}}$, which have been calculated using the following relation

$$
\nabla \cdot \overline{\mathbf{q}}=4 \pi \bar{\kappa} \bar{I}_{b}-\int_{4 \pi} \bar{\kappa} \bar{I} d \Omega .
$$

In Eq. (34), the first term on the right hand side represents the emitted/outgoing radiation from a computational control volume, while the second term represents the total incident radiation into that control volume. Thus, $\nabla . \overline{\mathbf{q}}$ gives the rate of the generation of energy by radiation and this must be coupled in the overall energy conservation. In this figure, the dashed lines represent the negative contours.

Fig. 8 shows the results of another important radiation property known as the incident radiation, $\bar{G}$, related to the radiative energy density, by which the total radiation energy is stored in each computational node and it is defined as

$$
\bar{G}=\int_{4 \pi} \bar{I} d \Omega \approx \sum_{m=1}^{M} \omega_{m} \bar{I}_{m} .
$$

The radial profiles of $\bar{G}$ have been plotted in Fig. 8 at various different locations on the mid-horizontal plane and show a distinct variation of the energy storage 
inside the combustion chamber. This figure shows that the value of $\bar{G}$ is lower in the nozzle region (see for $y=165 \mathrm{~mm}$ ) due to the effect of the large amount of air flowing through the Dilution ports for cooling; no combustion occurs downstream of these ports. The rate of the radiative absorption-emission in that region is also lower as both the flame temperature and the concentrations of $\mathrm{H}_{2} \mathrm{O}$ and $\mathrm{CO}_{2}$ are predicted to be lower (see in Fig. 6v). However, inside the combustor barrel (at $y=130 \mathrm{~mm}$, $95 \mathrm{~mm}$ and $50 \mathrm{~mm}), \bar{G}$ attains a maximum as this part of the combustor houses the extremely hot gases, though in the head of the combustor (at $y=20 \mathrm{~mm}$ ) $\bar{G}$ is lower. The prediction of the incident radiation is an essential task which allows the radiative energy transfer to be coupled with global energy conservation (for example, see Eq. (34)).

The predictions of the net wall radiative heat fluxes, $\overline{\mathbf{q}}_{n}=\epsilon_{w}\left(\overline{\mathbf{q}}_{i n}-\pi \bar{I}_{b w}\right)$, on the inlet, the outlet and the four different horizontal surfaces of the combustor are shown in Fig. 9. In this figure, the solid lines indicate the positive contours while the dashed lines indicate the negative contours. At most places of the combustor barrel, the liner walls lose heat by radiative transfer, but gain heat in both head and nozzle areas (see Fig. 9c-f). Also, the combustor surfaces gain heat in the vicinity of the Primary and the Dilution ports.

\section{Conclusion}

The $S_{4}$ approximation of the discrete ordinate method has been implemented to investigate the radiative heat transfer inside a model gas turbine combustor. The DOM has been combined with a Large Eddy Simulation of the flow, temperature and composition fields within the combustion chamber. A gray-gas approximation to the RTE has been assumed (i.e., the transmission of the radiative intensities is independent of the wavelength). The absorption coefficient for both $\mathrm{H}_{2} \mathrm{O}$ and $\mathrm{CO}_{2}$ gases is calculated but scattering effects are neglected in the present work.

The instantaneous values of the radiation properties such as the radiative heat fluxes, the incident radiation, the energy source or sink as the divergence of radiative heat fluxes $(\nabla \cdot \overline{\mathbf{q}})$ have been calculated. The net radiative heat fluxes on the liner walls of the combustor have also been calculated. A coupling of this radiative heat gain or loss is likely to yield accurately predicted wall temperature and this will aid combustor design by allowing an optimum amount of air to be used for wall cooling. The beneficial effects will be a reduction in the emission of pollutant gases by maximising the combustion efficiency and to allow a longer liner life.

The present study excludes the effects of the soot on radiative heat transfer. Soot is likely to enhance the radiation field and ultimately the coupling of soot formation and consumption to heat radiation is an important requirement. The conservation of soot concentrations is required to measure the soot properties and Research is currently underway on this.

Acknowledgements: The authors would like to acknowledge the EPSRC for financial support for this project. The second author thanks Prof. T. Alexander of the Department of Mechanical Engineering, University of Glasgow for allowing him 
an access to a dual processor LINUX machine.

\section{References}

[1] S. Chandrasekhar, Radiative Transfer, Dover Publications, 1960.

[2] B. G. Carlson, K. D. Lathrop, Transport theory-the method of discrete ordinates, in: Computing Methods in Reactor Physics, Gordon and Breach, New York, 1968, pp. 165-266.

[3] W. A. Fiveland, Three-dimensional radiative heat-transfer solutions by the discrete-ordinates method, J. Thermophys. 2(4) (1988) 309-316.

[4] A. S. Jamaluddin, P. J. Smith, Predicting radiative transfer in rectangular enclosures using the discrete ordinates method, Comb. Sci. and Tech. 59 (1988) $321-340$.

[5] J. C. Chai, S. V. Patankar, H. S. Lee, Evaluation of spatial differencing practices for the discrete-ordinates method, J. Thermophys. and Heat Tran. 8(1) (1994) $140-144$.

[6] J. Liu, H. M. Shang, Y. S. Chen, T. S. Wang, Prediction of radiative transfer in general body-fitted coordinates, Num. Heat Tran. Part B 31 (1997) 423-439.

[7] N. Kayakol, N. Selcuk, I. Campbell, O. L. Gulder, Performance of discrete ordinates method in a gas turbine combustor simulator, Exp. Thermal and Fluid Sci. 21 (2000) 134-141.

[8] C. R. Kaplan, S. W. Baek, E. S. Oran, J. L. Ellzey, Dynamics of a strongly radiating unsteady ethylene jet diffusion flame, Combustion and Flame 96 (1994) $1-21$.

[9] L. H. Howell, R. B. Pember, P. Colella, J. P. Jessee, W. A. Fiveland, A conservative adaptive-mesh algorithm for unsteady, combined-mode heat transfer using the discrete ordinates method, Num. Heat Tran. Part B 35 (1999) 407-430.

[10] P. E. Desjardin, S. H. Frankel, Two-dimensional large eddy simulation of soot formation in the near-field of a strongly radiating nonpremixed acetylene-air turbulent jet flame, Combustion and Flame 119 (1999) 121-132.

[11] S. P. Burns, Review of radiative transfer models for large scale fire modelling using domain based parallelism, Tech. rep., Sandia National Laboratories, Albuquerque, New Mexico 87185-0835 (1996).

[12] F. di Mare, Radiative heat transfer: an overview, Tech. rep., Imperial College London (2002).

[13] U. Piomelli, Large-eddy simulation: achievements and challenges, Progress in Aerospace Sciences 35 (1999) 335-362. 
[14] W. P. Jones, Large eddy simulation of turbulent combustion processes, Comp. Phys. Coms. 147 (2002) 533-537.

[15] F. di Mare, W. P. Jones, K. Menzies, Large eddy simulation of a model gas turbine combustor, Combustion and Flame 137 (2004) 278-294.

[16] A. F. Bicen, D. G. N. Tse, J. H. Whitelaw, Combustion characteristics of a model can-type combustor, Tech. rep. fs/87/28, Imperial College London (1987).

[17] A. Leonard, Energy cascade in large-eddy simulations of turbulent fluid flows, Advances in Geophysics 18A (1974) 237-248.

[18] A. Favre, Statistical equations of turbulent cases in problems of hydrodynamics and continuum mechanics, Tech. rep., Society of Industrial and Applied Mathematics, Philadelphia (1969).

[19] M. F. Modest, Radiative heat transfer, second edition, Academic Press, 2003.

[20] M. Germano, A proposal for a redefinition of the turbulent stresses in the filtered navier-stokes equations, Phys. of Fluids 29(7) (1986) 2323-24.

[21] J. Smagorinsky, General circulation experiments with the primitive equations. i. the basic experiment, Monthly Weather Review 91 (1963) 99-164.

[22] H. Schmidt, U. Schumann, Coherent structure of the convective boundary layer derived from large eddy simulation, J. Fluid Mech. 200 (1989) 511-562.

[23] A. H. Lefebvre, Flame radiation in gas turbine combustion chambers, Int. J. Heat Mass Tran. 27(9) (1984) 1493-1510.

[24] B. F. Magnussen, B. H. Hjertager, On mathematical modelling of turbulent combustion with special emphasis on soot formation and combustion, in: 16th Symposium (International) on Combustion, The Combustion Institute, 1976, pp. 719-729.

[25] J. F. Thompson, F. Thames, C. Mastin, Automatic numerical generation of body-fitted curvilinear coordinates system for field containing any number of arbitrary two-dimensional bodies, J. Comp. Phys. 15 (1974) 299-319.

[26] N. Branley, W. P. Jones, Large eddy simulation of a turbulent nonpremixed flame, Combustion and Flame 127 (2001) 1913-1934.

[27] F. di Mare, Large eddy simulation of reacting and non-reacting turbulent flows, Ph.D. thesis, Imperial College London (2002).

[28] K. D. Lathrop, Spatial differencing the transport equation: positivity vs. accuracy, J. Comp. Phys. 4 (1969) 475-498.

[29] J. C. Chai, G. Parthasarathy, H. S. Lee, S. V. Patankar, Finite volume radiative heat transfer procedure for irregular geometries, J. Thermophys. Heat Tran. 9 (1995) 410-415. 
[30] M. Menguc, R. Viskanta, Radiative transfer in three-dimensional rectangular enclosures, J. Quan. Spec. Rad. Tran. 33 (1985) 533-549. 


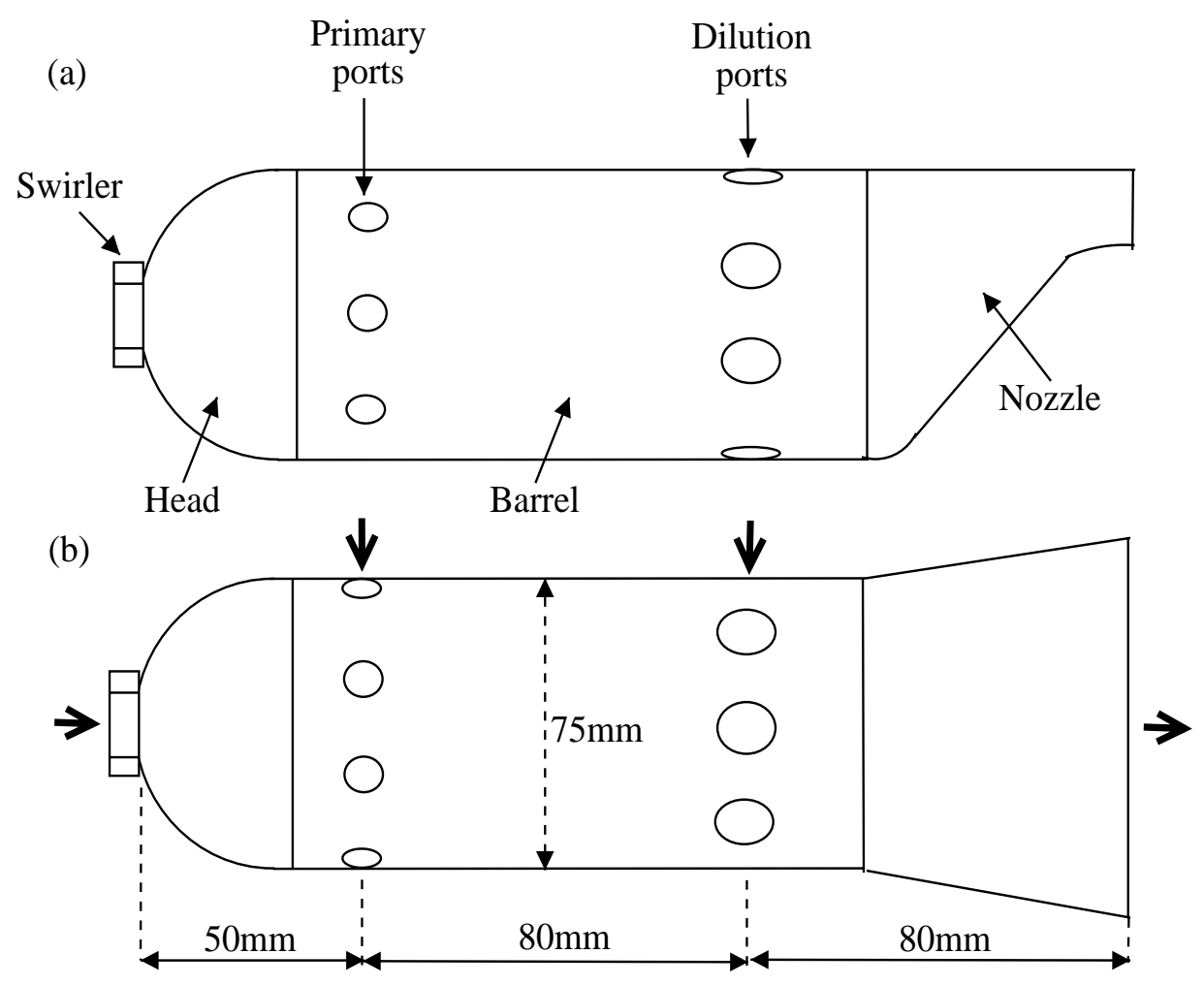

Figure 1: The features of the can-type model Tay gas turbine combustor; (a) vertical (on $y$ - $x$ plane) and (b) horizontal (on $y$-z plane) positions. 


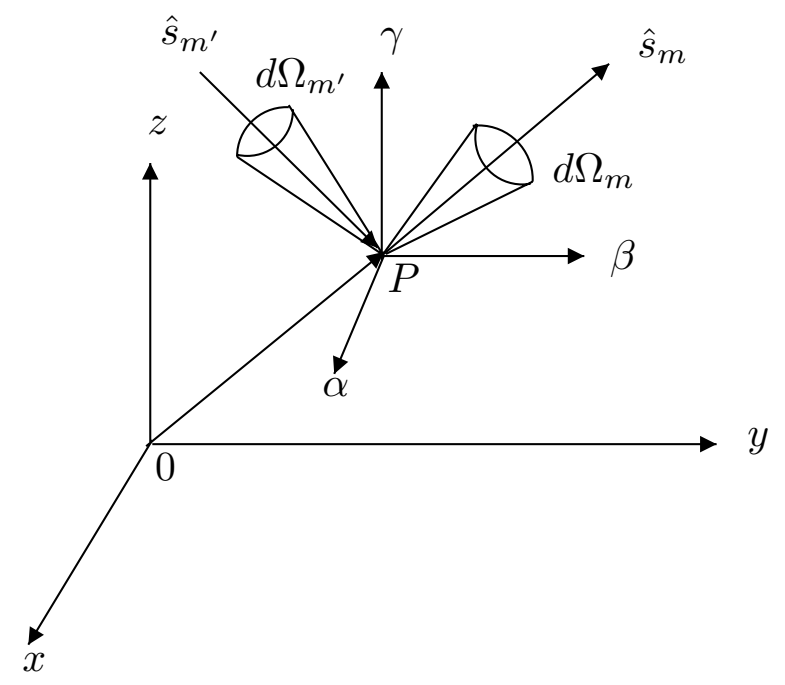

Figure 2: Representation of the angular coordinates and the solid angles $\left(d \Omega_{m}\right.$, $\left.d \Omega_{m^{\prime}}\right)$ along incoming $\left(\hat{s}_{m^{\prime}}\right)$ and outgoing $\left(\hat{s}_{m}\right)$ directions. 


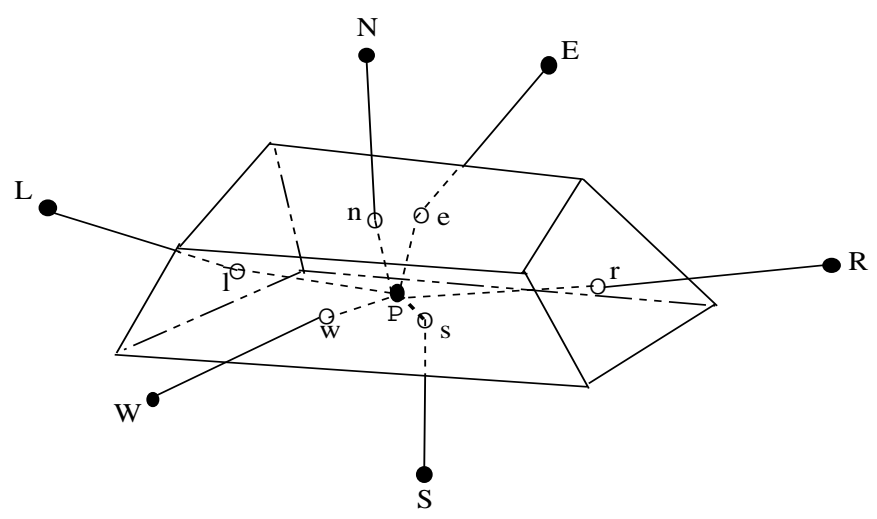

Figure 3: A representative control volume; solid circles indicate the node points while hollow circles indicate the surface nodes. 


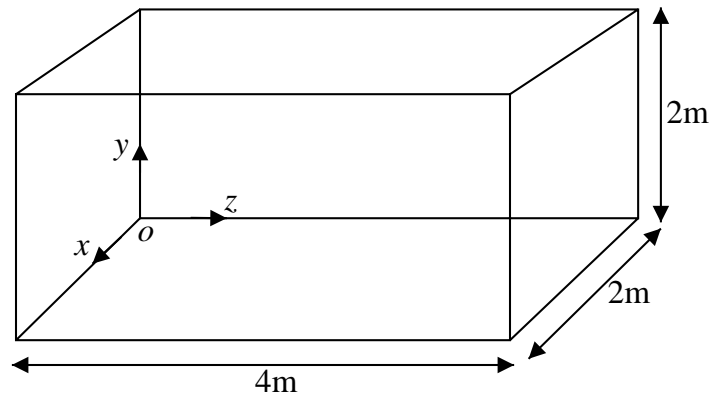

(a)

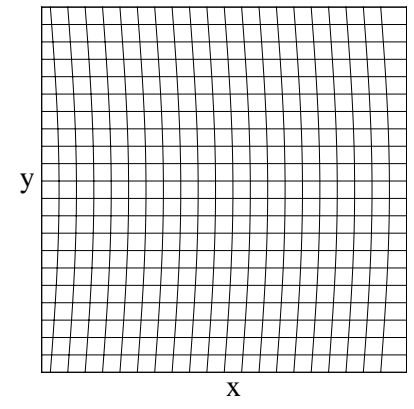

(b)

Figure 4: An idealised rectangular furnace, (a) schematic and (b) non-orthogonal grid. 

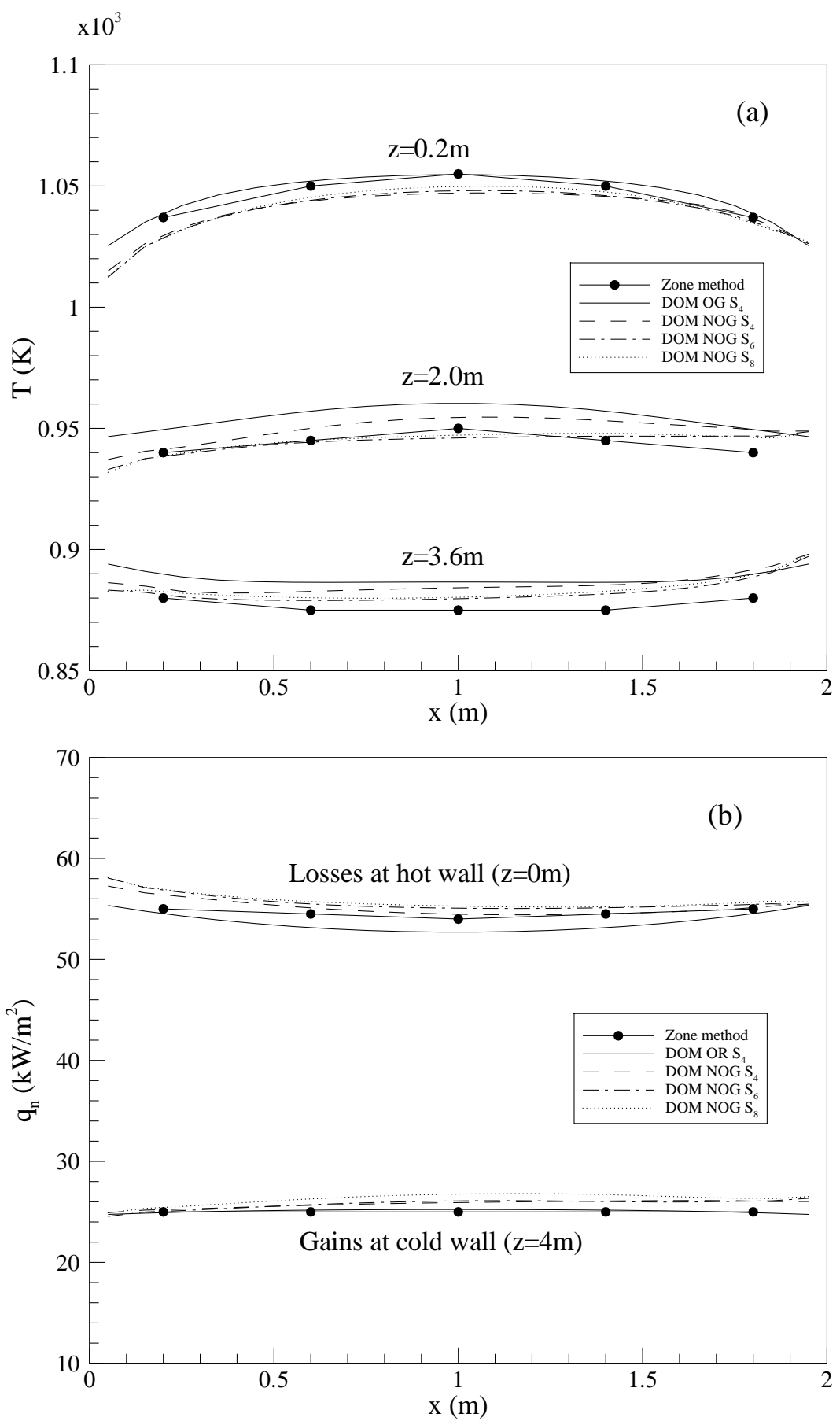

Figure 5: Comparison of the DOM non-orthogonal grid (NOR) results with others for (a) temperature at three $z$ locations and (b) wall radiative heat flux distributions at the hot and cold walls. 
a

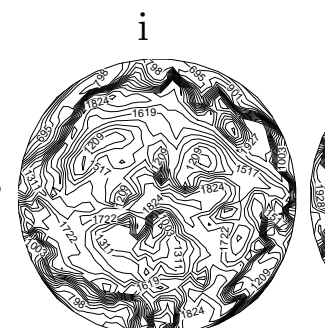

$\mathrm{b}$

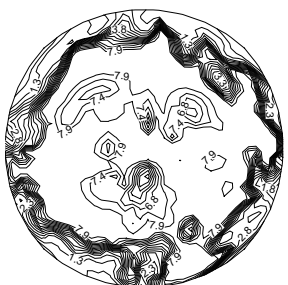

$\mathrm{c}$

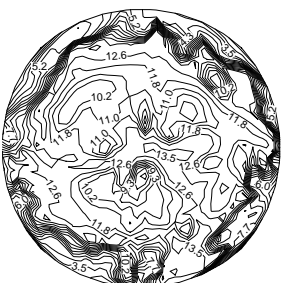

d
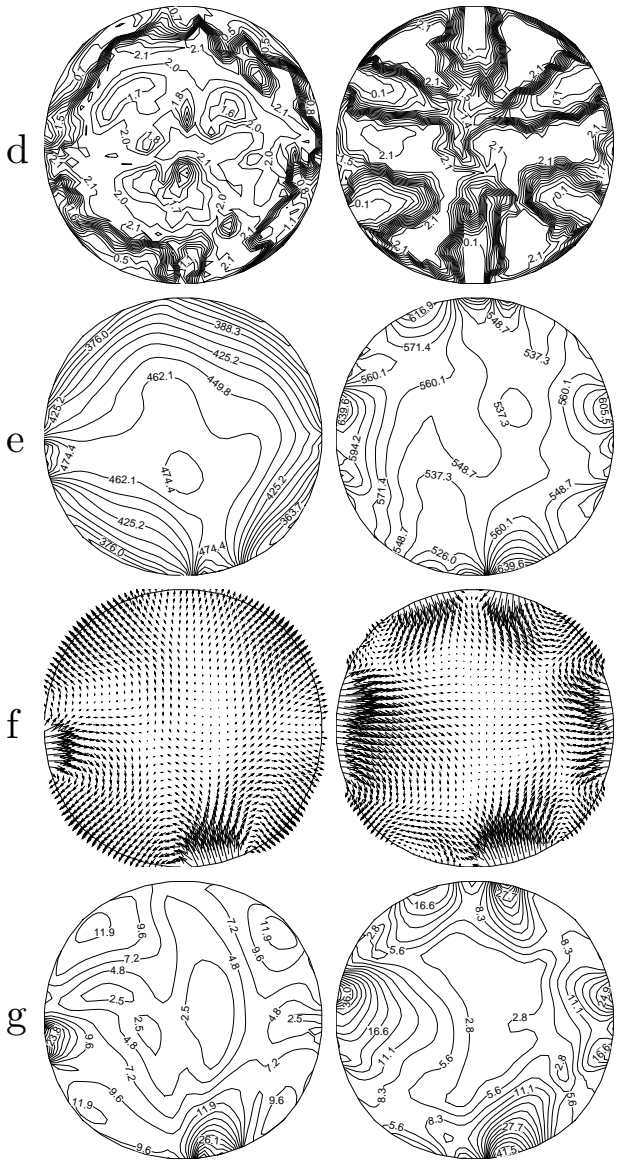
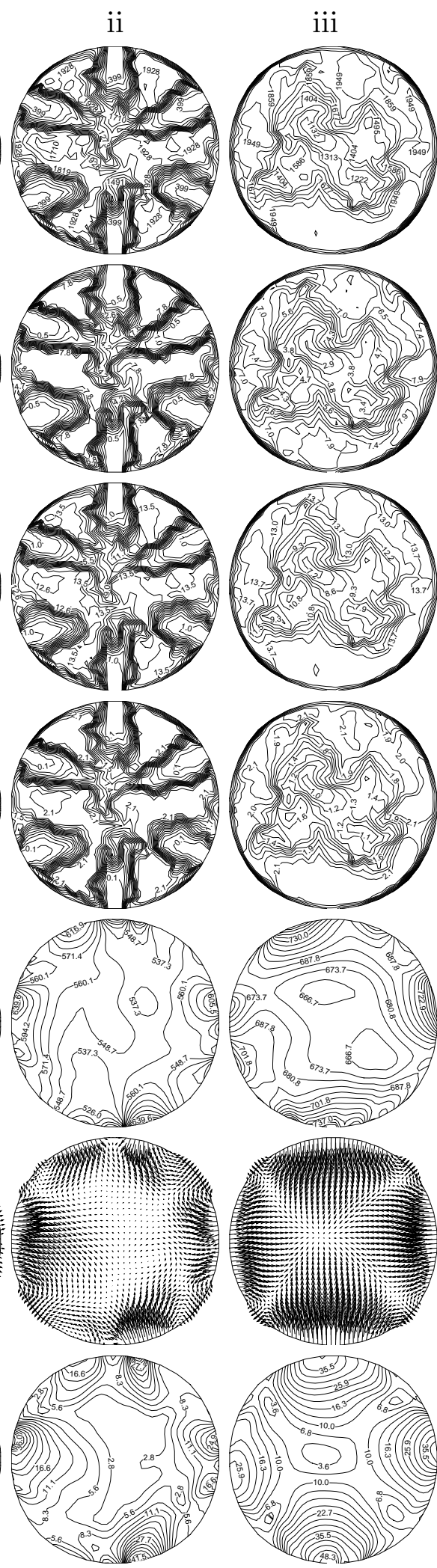
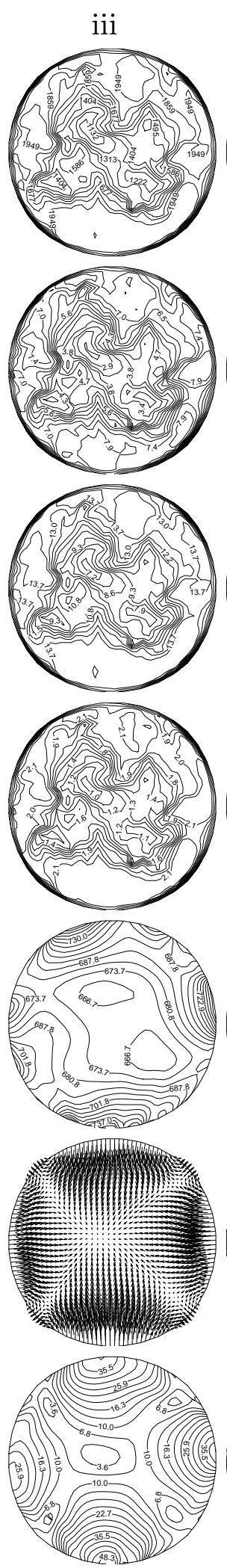
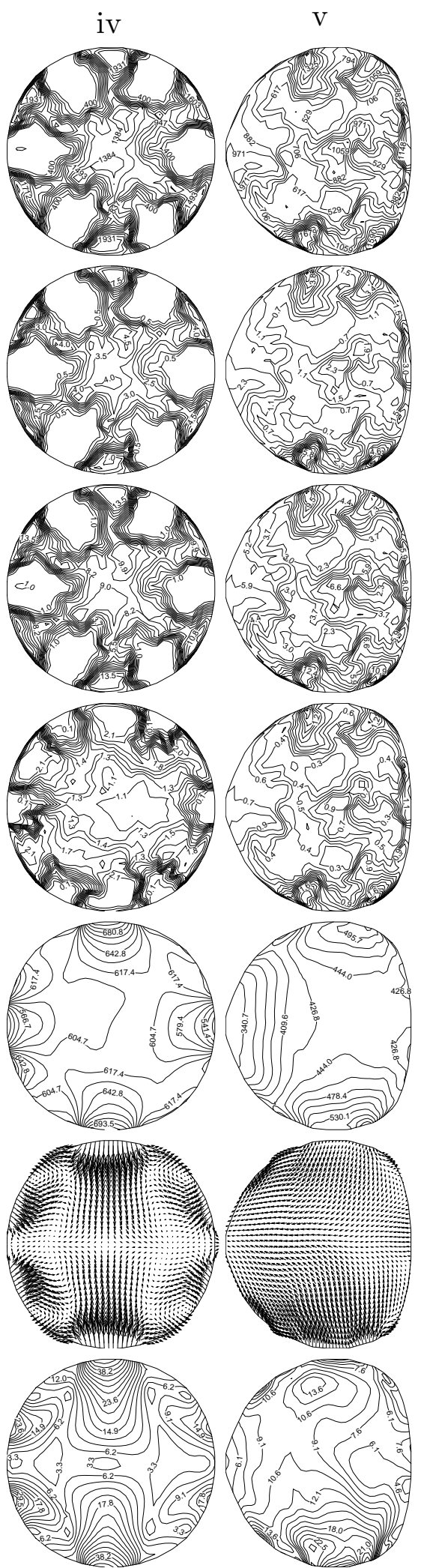

Figure 6: Instantaneous contour plots of (a) $\tilde{T}(K)$, (b) $\tilde{Y}_{\mathrm{CO}_{2}} \times 10^{-2}$, (c) $\tilde{Y}_{\mathrm{H}_{2} \mathrm{O}} \times$ $10^{-2}$, (d) $\bar{\kappa}\left(\mathrm{cm}^{-1}\right)$, (e) $\bar{I}\left(k W m^{-2}\right)$, (f) $\overline{\mathbf{q}}$ and (g) $|\overline{\mathbf{q}}|\left(k W m^{-2}\right)$ at various horizontal positions, (i) $y=20 \mathrm{~mm}$, (ii) $y=50 \mathrm{~mm}$, (iii) $y=95 \mathrm{~mm}$, (iv) $y=130 \mathrm{~mm}$ and (iv) $y=165 \mathrm{~mm}$, of the combustor. 


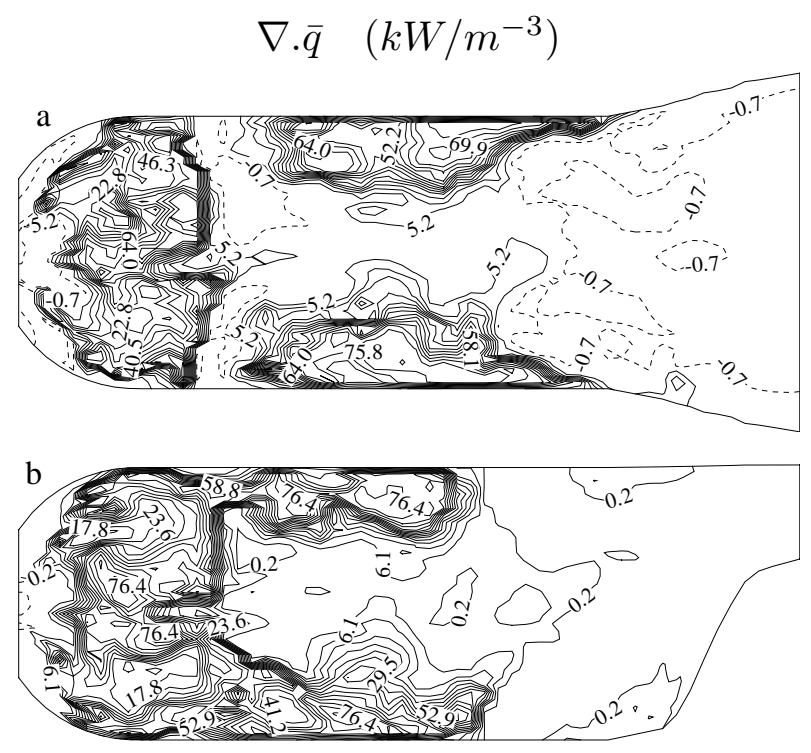

Figure 7: Contours of the divergence of the radiative heat fluxes on (a) the midhorizontal and (b) the mid-vertical planes of the combustor. 


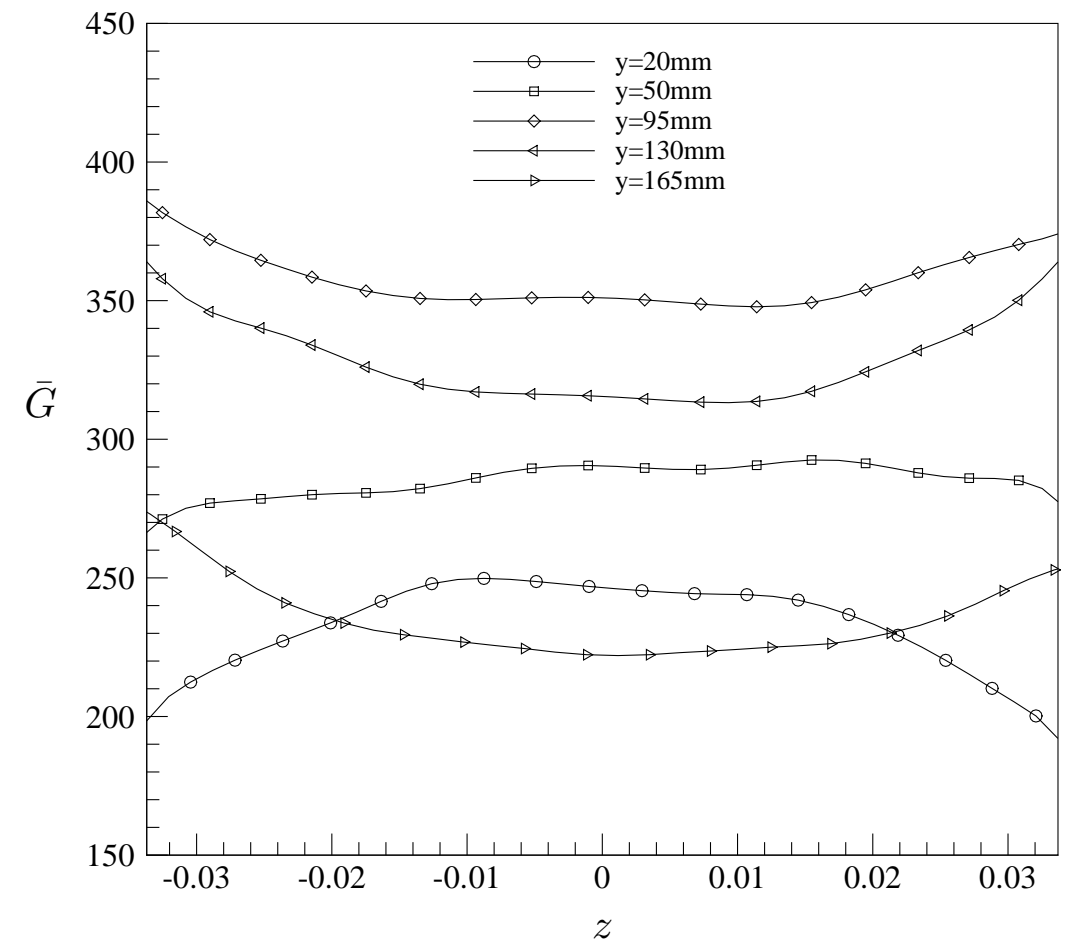

Figure 8: Radial profiles of the incident radiation, $\bar{G}\left(\mathrm{kWm}^{-2}\right)$, at various locations on the mid-horizontal plane of the combustor. 

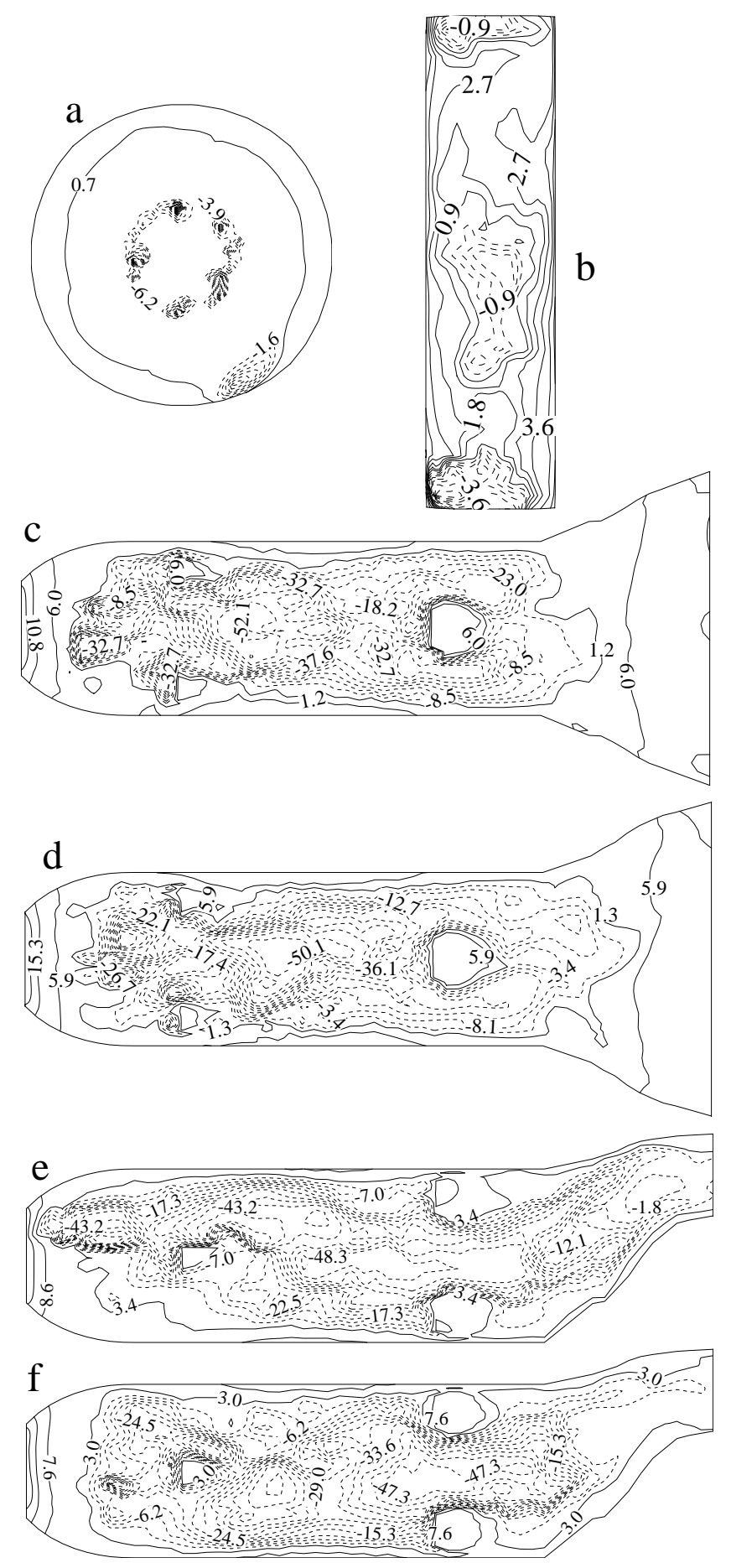

Figure 9: The net radiative heat fluxes, $\overline{\mathbf{q}}_{n}\left(k W \mathrm{~m}^{-2}\right)$, on the liner walls of the combustor; here (a) $y=0$, (b) $y=L_{y}$, (c) $x=-L_{x} / 2$, (d) $x=L_{x} / 2$, (e) $z=-L_{z} / 2$, and (f) $z=L_{z} / 2$. 


\begin{tabular}{ccccc}
\hline $\begin{array}{c}S_{n} \\
\text { Approximation }\end{array}$ & $\alpha_{m}$ & $\begin{array}{c}\text { Ordinates } \\
\beta_{m}\end{array}$ & $\begin{array}{c}\gamma_{m} \\
\omega_{m}\end{array}$ \\
\hline$S_{4}$ & 0.2958759 & 0.2958759 & 0.9082483 & 0.5235987 \\
& 0.2958759 & 0.9082483 & 0.2958759 & 0.5235987 \\
& 0.9082483 & 0.2958759 & 0.2958759 & 0.5235987 \\
\hline$S_{6}$ & 0.1838670 & 0.1838670 & 0.9656013 & 0.1609517 \\
& 0.1838670 & 0.6950514 & 0.6950514 & 0.3626469 \\
& 0.1838670 & 0.9656013 & 0.1838670 & 0.1609517 \\
& 0.6950514 & 0.1838670 & 0.6950514 & 0.3626469 \\
& 0.6950514 & 0.6950514 & 0.1838670 & 0.3626469 \\
$S_{8}$ & 0.9656013 & 0.1838670 & 0.1838670 & 0.1609517 \\
\hline & 0.1422555 & 0.1422555 & 0.9795543 & 0.1712359 \\
& 0.1422555 & 0.5773503 & 0.8040087 & 0.0992284 \\
& 0.1422555 & 0.8040087 & 0.5773503 & 0.0992284 \\
& 0.1422555 & 0.9795543 & 0.1422555 & 0.1712359 \\
& 0.5773503 & 0.1422555 & 0.8040087 & 0.0992284 \\
& 0.5773503 & 0.5773503 & 0.5773503 & 0.4617179 \\
& 0.5773503 & 0.8040087 & 0.1422555 & 0.0992284 \\
& 0.8040087 & 0.1422555 & 0.5773503 & 0.0992284 \\
& 0.8040087 & 0.5773503 & 0.1422555 & 0.0992284 \\
& 0.9795543 & 0.1422555 & 0.1422555 & 0.1712359 \\
\hline
\end{tabular}

Table 1: Discrete ordinates for the $S_{n}$ approximation $[2 ; 19]$ for the first quadrant. 


\begin{tabular}{cc}
\hline Parameters & Values \\
\hline step size, $d t$ & $5.53 \times 10^{-7}(\mathrm{sec})$ \\
Smagorinsky constant, $C_{s}$ & 0.1 \\
emissivity of walls, $\epsilon_{w}$ & 0.1 \\
Stefan-Boltzmann constant, $\sigma$ & $5.67 \times 10^{-8}\left(\mathrm{Wm}^{-2} \mathrm{~K}^{-4}\right)$ \\
\hline
\end{tabular}

Table 2: Computational parameters. 